BULL. AUSTRAL. MATH. SOC.

VOL. I (1969), 315-317.

\title{
Topologies on finite groups
}

\section{Sidney A. Morris and H. B. Thompson}

\begin{abstract}
It has been shown by D. Stephen that the number $N$ of open sets in a non-discrete topology on a finite set with $n$ elements is not greater than $3 \times 2^{n-2}$. We show that for admissable topologies on a finite group $N \leqq 2^{n / r}$, where $r$ is the least order of its non-trivial normal subgroups. This is clearly a sharper bound.
\end{abstract}

Define semi-topological group and topological group as in [2]. (We do not assume that these topological spaces are Hausdorff.)

THEOREM 1. Let $X$ be a non-discrete finite semi-topological group of order $n(>1)$. Then the number $N$ of open sets satisfies $N \leqq 2^{n / r}$, where $r$ is the least order of the non-trivial normal subgroups of $X$.

Proof. Let $0_{x}$ be the intersection of all open sets containing $x \in X$. Because there are only a finite number of open sets $o_{x}$ is open. We show that $0_{e}$ (where $e$ is the identity of $X$ ) is a normal subgroup. Since, for each $x \in X, x_{e}$ and $x^{-1} 0_{x}$ are open (Theorem 1 , Chapter II of [2]) and contain $x$ and $e$ respectively, $0_{x} \subseteq x 0_{e}$ and $0_{e} \subseteq x^{-1} o_{x}$ which imply

$$
0_{x}=x 0_{e} .
$$

Similarly

Received 28 April 1969. This paper was written while the authors held Research Assistantships at the University of Queensland. The authors greatly appreciate the suggestions made by Mr M.P. O'Donnell and Professor R. Výborný. 


$$
{ }_{x}^{0}=e^{x}
$$

Clearly if $y \in 0_{e}, o_{e} \supseteq 0_{y}=y 0_{e}$. (In fact since $o_{e}$ and $y 0_{e}$ have the same finite number of elements $o_{e}=y 0_{e}$. ) Thus

$$
0_{e}^{2} \subseteq 0_{e}
$$

Since $X$ is a finite group and ${ }_{e}$ is non-empty, (1), (2) and (3) imply $o_{e}$ is a normal subgroup.

The $0_{x}$ are the cosets of $0_{e}$. Indeed $\left\{0_{e}\right\}$ is an open basis at $e$ for the topology. Thus the number of distinct $0_{x}$ is $\frac{n}{m}$, where $m$ is the order of $0_{e}$. (Since the topology was assumed to be non-discrete $m$ is strictly greater than one.) The proof is completed by noting that the number of open sets is $2^{n / m} \leqq 2^{n / r}$. (See [3]).

The following example shows that the bound in the above theorem is the best possible (under the conditions of the theorem).

EXAMPLE. Let $X$ be the additive group of integers $\bmod n$ and $o_{e}=\left\{0, \frac{n}{p}, \frac{2 n}{p}, \ldots, \frac{(p-1) n}{p}\right\}$, where $p$ is the smallest prime dividing $n$. By Theorem 4.5 of [1], the topology on $X$ which has $\left\{0_{e}\right\}$ as an open basis at 0 makes $X$ a topological group. It is obvious that the number of open sets is $2^{n / p}$.

COROLLARY 2. Every finite semi-topological group is a topological group.

Proof. By Theorem $1 o_{e}$ is a normal subgroup and $\left\{0_{e}\right\}$ is an open basis for the topology. The result now follows from Theorem 4.5 of [1].

For completeness we include the following remark.

REMARK. It is easily shown that if there is a topology on a group $X$ (not necessarily finite) such that the mapping $(x, y) \rightarrow x^{-1} y$ is continuous in the first variable then $(x, y) \rightarrow x y$ is continuous in each variable separately and $x+x^{-1}$ is continuous. If $X$ is also finite then by Corollary $2, X$ is a topological group. 


\section{References}

[1] Edwin Hewitt and Kenneth A. Ross, Abstract harmonic analysis, Vol. 1, (Academic Press, New York, 1963).

[2] Taqdir Husain, Introduction to topological groups, (W.B. Saunders Company, Philadelphia and London, 1966).

[3] D. Stephen, "Topologies on finite sets", Amer. Math. Monthly 75 (1968), 739-741.

The Flinders University of South Australia.

The University of Queensland. 